SHORT REPORT

\title{
The significance of a high plasma ammonia value
}

\section{S L Chow, V Gandhi, S Krywawych, P T Clayton, J V Leonard, A A M Morris}

At a tertiary referral centre, just over $50 \%$ of patients with plasma ammonia values over $200 \mu \mathrm{mol} / \mathrm{l}$ had inborn errors of metabolism. To distinguish artefactual high values from those requiring treatment, the measurement should be repeated immediately if the result is above $200 \mu \mathrm{mol} / \mathrm{l}$ and at lower concentrations if the patient is encephalopathic.

$\mathrm{T}$ he measurement of plasma ammonia is an important screening investigation for many inborn errors of metabolism, but mild to moderate hyperammonaemia can occur as a non-specific finding in sick children, particularly neonates. Increased plasma ammonia values can also result from artefact if the sample is taken or processed incorrectly. ${ }^{1}$ This can make the interpretation difficult. Plasma ammonia concentrations above $200 \mu \mathrm{mol} / \mathrm{l}$, however, are generally thought to be highly suggestive of an inborn error of metabolism. To see if this is correct, we have reviewed 90 children who had ammonia values above $200 \mu \mathrm{mol} / \mathrm{l}$ in a tertiary referral hospital.

\section{METHODS}

At Great Ormond Street Hospital, London, plasma ammonia concentrations are determined by the VITROS AMON 700 slide system. ${ }^{2}$ Specimens are not transported to the laboratory on ice but are sent by vacuum tube, which should minimise delay and the risk of artefactual rises in ammonia concentration. In the laboratory, if the specimen is not analysed at once, it is separated and frozen. Haemolysis increases plasma ammonia concentration; this is always reported in our results and a repeat specimen is requested.

In this study, plasma ammonia measurements were reviewed for the 10 year period 1992-2002. For patients with values above $200 \mu \mathrm{mol} / \mathrm{l}$, the medical records were retrieved. Additional details were recorded, including the patient's condition at the time of the high ammonia value, additional plasma ammonia measurements, other metabolic investigations, and the final diagnosis.

\section{RESULTS}

During the study period, plasma ammonia was measured on 9958 occasions. Of these, 394 values from 126 patients were greater than $200 \mu \mathrm{mol} / \mathrm{l}$. We reviewed the records of 90 patients; the records of the other patients could not be retrieved.

Table 1 shows the causes of hyperammonaemia in the 90 patients. Figure l shows ammonia concentrations for patients grouped according to the underlying cause; for patients who had more than one ammonia measurement, the highest value is shown. Altogether, 54\% of the patients had an inborn error of metabolism. As expected, most of these had urea cycle defects; the next largest group were the branched chain organic acidaemias. Four patients had acute liver failure due to tyrosinaemia type 1. Only one patient had a fatty acid oxidation disorder, although some of these disorders are common in the UK and they can cause hyperammonaemia during acute decompensation. The low frequency in our study probably reflects the specialist nature of the hospital; hyperammonaemia in fatty acid oxidation disorders may have resolved by the time patients reach this hospital.

Of the 41 patients who did not have an inborn error, three had acute liver failure and two had transient hyperammonaemia of the newborn. ${ }^{3}$ For the remaining 36 patients, no cause could be found for the high plasma ammonia value. In 17 patients, the plasma ammonia concentration was less than $60 \mu \mathrm{mol} / \mathrm{l}$ when repeated, without any treatment having been given. We suspect that the high ammonia values were artefactual in many of these cases, although some patients may have had transiently raised concentrations (for example, due to severe illness). In 19 patients, ammonia measurements were not repeated. Some of these high ammonia

Table 1 Causes of hyperammonaemia according to age in this cohort of patients

\begin{tabular}{lllll}
\hline & Age & & & \\
\cline { 2 - 5 } & $<1$ mth & 1-12 mth & 1-16 y & Total \\
\hline Urea cycle defects & 17 & 6 & 5 & 28 \\
Branched chain organic acidaemias & 9 & 0 & 0 & 9 \\
Tyrosinaemia type 1 (with liver failure) & 0 & 3 & 1 & 4 \\
Congenital lactic acidoses & 1 & 2 & 0 & 3 \\
Hyperinsulinism with hyperammonaemia & 1 & 0 & 1 & 2 \\
Carnitine transporter defect & 0 & 1 & 0 & 1 \\
Glutaric aciduria type I & 0 & 0 & 1 & 1 \\
Congenital defect of glycosylation & 0 & 0 & 1 & 1 \\
Transient hyperammonaemia of the newborn & 2 & 0 & 0 & 2 \\
Liver failure (without inborn error) & 0 & 1 & 2 & 3 \\
Unexplained & & & 7 & 17 \\
$\quad$ Repeat ammonia normal & 9 & 1 & 7 & 6 \\
Patient died (no repeat measurement) & 3 & 3 & 0 & 13 \\
No repeat measurement & 5 & 3 & 5 & \\
\hline Results expressed as number of cases. & & & & \\
\end{tabular}




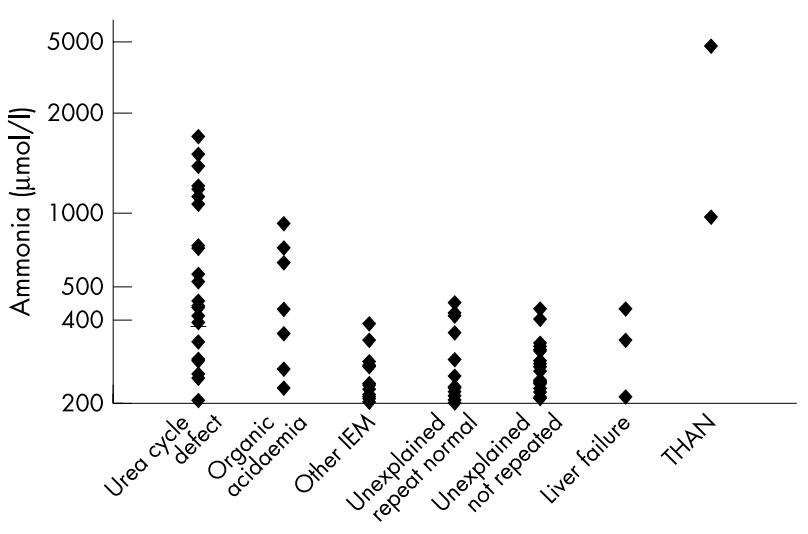

Figure 1 Plasma ammonia concentrations by diagnosis. Concentrations have been plotted on a logarithmic scale. IEM, inborn errors of metabolism; THAN, transient hyperammonaemia of the newborn.

values were probably artefactual, but obviously this is speculative. In six of the 19 cases, the ammonia measurement was not repeated because the patient died soon after the high value, the causes of death being sepsis in three, respiratory distress syndrome in two, and congenital heart disease in one child. Investigations to exclude inborn errors were undertaken in three of the six patients who died and, for the others, review of the notes did not reveal any pointers to an inborn error. Thus, in these patients the high ammonia values $(242-352 \mu \mathrm{mol} / \mathrm{l})$ may have been a non-specific effect of severe illness. Of the 13 patients who had a single ammonia measurement and survived, only seven had investigations to exclude inborn errors; again, review of the notes in the others did not reveal any pointers to metabolic disease.

\section{DISCUSSION}

Great Ormond Street Hospital, London, is a tertiary referral children's hospital with neonatal, paediatric, and cardiothoracic intensive care units and a large metabolic unit but no liver transplant unit. The specialist nature of the hospital will have influenced the frequency of hyperammonaemia and the pattern of causes. This study could not, however, have been undertaken elsewhere, because a less specialised hospital would not have enough cases of severe hyperammonaemia to evaluate.

The study shows that plasma ammonia values above $200 \mu \mathrm{mol} / \mathrm{l}$ are rare. Even in this hospital, only $4 \%$ of plasma ammonia measurements were above this concentration. The proportion would probably be much lower in most other hospitals, where there are unlikely to be so many metabolic patients or sick intensive care unit patients.

In this study, inborn errors account for just over half of the plasma ammonia values above $200 \mu \mathrm{mol} / \mathrm{l}$. A few of the patients with unexplained hyperammonaemia may have had unrecognised inborn errors, but it seems unlikely that many of them did. In liver transplant centres, one might expect liver failure to account for a higher proportion of cases of hyperammonaemia. Our study suggests, however, that a considerable proportion of high ammonia values may be artefactual, even if appropriate precautions are taken.

Almost all patients with a plasma ammonia concentration above $500 \mu \mathrm{mol} / \mathrm{l}$ had an inborn error of metabolism (fig 1). Ammonia concentrations above $200 \mu \mathrm{mol} / \mathrm{l}$ were less specific for inborn errors and the specificity did not improve significantly until the threshold reached $500 \mu \mathrm{mol} / \mathrm{l}$.

Plasma ammonia measurement should be repeated whenever a raised concentration is reported, because even mild hyperammonaemia can be a pointer to metabolic disease. Moderate or severe hyperammonaemia is highly toxic to the brain. A repeat specimen should, therefore, be sent immediately if the reported concentration is above $200 \mu \mathrm{mol} / \mathrm{l}$ or if the patient is encephalopathic. We suggest that patients with a plasma ammonia value above $200 \mu \mathrm{mol} / \mathrm{l}$ should be regarded as having an inborn error until proven otherwise, although the specificity may be only around $50 \%$. Even before the repeat result is available, a metabolic centre should be contacted with a view to lowering the ammonia concentration.

\section{Authors' affiliations}

S L Chow, V Gandhi, S Krywawych, P T Clayton, J V Leonard, A A M Morris, Metabolic Unit, Great Ormond Street Hospital, London WCIN 3JH, UK

Correspondence to: Dr A Morris, Willink Biochemical Genetics Unit, Royal Manchester Children's Hospital, Hospital Road, Pendlebury, Manchester M27 4HA, UK; Andrew.Morris@cmmc.nhs.uk

Accepted 8 October 2003

\section{REFERENCES}

1 Barsotti RJ. Measurement of ammonia in blood. J Pediatr 2001; 138(suppl 1):S1 1-20.

2 Sundberg MW, Becker RW, Esders TW, et al. An enzymic creatinine assay and a direct ammonia assay in coated thin films. Clin Chem 1983;29:645-9. 3 Hudak ML, Jones MD Jr, Brusilow SW. Differentiation of transient

hyperammonemia of the newborn and urea cycle enzyme defects by clinical presentation. J Pediatr 1985;107:712-19. 